\title{
Modeling the center-to-limb variation of the Ca I 4227 Å line using FCHHT models
}

\author{
H. D. Supriya ${ }^{1}$, H. N. Smitha ${ }^{1}$, K. N. Nagendra ${ }^{1}$, J. O. Stenflo ${ }^{2,3}$, \\ M. Bianda ${ }^{3}$, B. Ravindra ${ }^{1}$, R. Ramelli ${ }^{3}$ and L. S. Anusha ${ }^{4}$ \\ ${ }^{1}$ Indian Institute of Astrophysics, Bangalore 560034, India \\ email: hdsupriya@iiap.res.in, smithahn@iiap.res.in, knn@iiap.res.in, \\ ravindra@iiap.res.in \\ ${ }^{2}$ Institute of Astronomy, ETH Zurich, CH-8093 Zurich, Switzerland \\ email: stenflo@astro.phys.ethz.ch \\ ${ }^{3}$ Istituto Ricerche Solari Locarno, Via Patocchi, CH-6605 Locarno-Monti, Switzerland \\ email: mbianda@irsol.ch, ramelli@irsol.ch \\ ${ }^{4}$ Max Planck Institut für Sonnesystemforschung, Justus-von-Liebig-Weg 3, \\ D-37077 Göttingen, Germany \\ email: bhasari@mps.mpg.de
}

\begin{abstract}
The Ca I $4227 \AA$ is a chromospheric line exhibiting the largest degree of linear polarization near the limb, in the visible spectrum of the Sun. Modeling the observations of the center-to-limb variations (CLV) of different lines in the Second Solar Spectrum helps to sample the height dependence of the magnetic field, as the observations made at different lines of sight sample different heights in the solar atmosphere. Supriya et al. (2014) attempted to simultaneously model the CLV of the $(I, Q / I)$ spectra of the Ca I $4227 \AA$ line using the standard 1-D FAL model atmospheres. They found that the standard FAL model atmospheres and also any appropriate combination of them, fail to simultaneously fit the observed Stokes $(I, Q / I)$ profiles at all the limb distances $(\mu)$ satisfying at the same time all the observational constraints. This failure of 1-D modeling approach can probably be overcome by using multi-dimensional modeling which is computationally expensive. To eliminate an even wider choice of 1-D models, we attempt here to simultaneously model the CLV of the $(I, Q / I)$ spectra using the FCHHT solar model atmospheres which are updated and recent versions of the FAL models. The details of our modeling efforts and the results are presented.
\end{abstract}

Keywords. line: formation, methods: numerical, polarization, radiative transfer, scattering, Sun: atmosphere

\section{Introduction}

The Ca I $4227 \AA$ line is one of the commonly observed and well studied lines in the Second Solar Spectrum (SSS). Interesting features exhibited by this line, like the spatial variations in the wing polarization of the $(Q / I, U / I)$ spectra along the spectrograph slit, has been well studied in several earlier works. An account of the previous studies done on this line is given in Supriya et al. (2014). Studying the center-to-limb variations (CLV) of different lines in the SSS helps us sample different heights in the solar atmosphere and understand the scattering physics to use it in a better way for diagnostics of solar atmosphere.

In Supriya et al. (2014) a detailed simultaneous modeling of the observed CLV of both $I$ and $Q / I$ profiles of the Ca I $4227 \AA$ line was done using standard FAL model atmospheres (Fontenla et al., 1993). They concluded that the standard FAL model atmospheres fail 


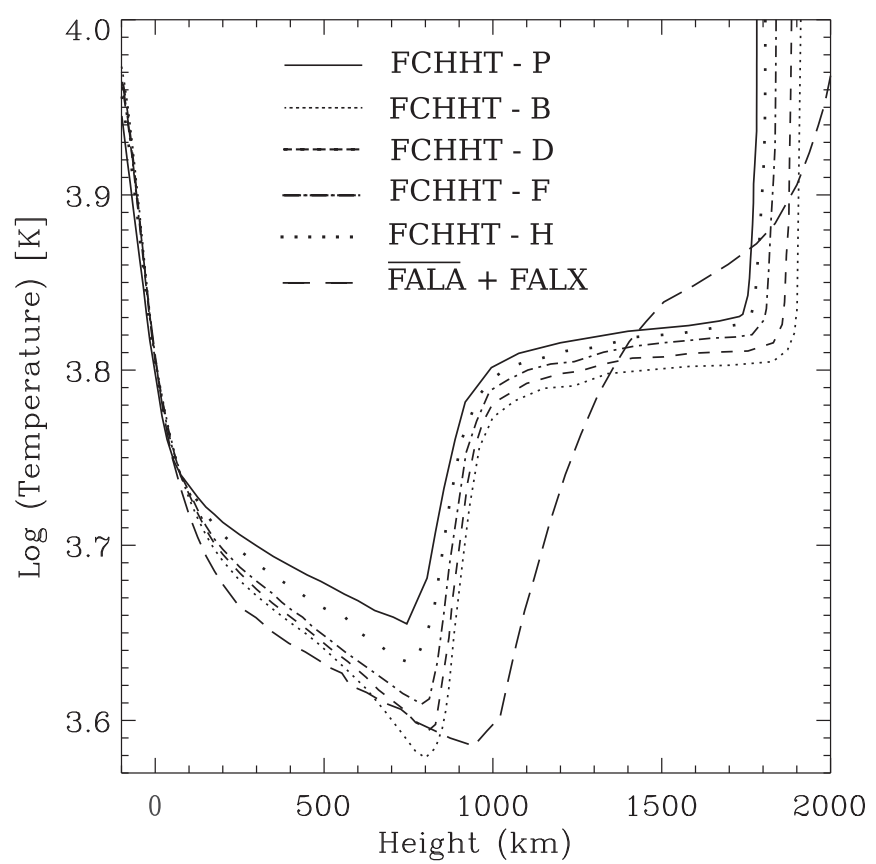

Figure 1. Temperature structure of the standard models FCHHT-B, FCHHT-D, FCHHT-F, FCHHT-H and FCHHT-P used in our studies. Along with these models the temperature structure of the combined model atmosphere $\overline{\text { FALA }}+$ FALX is also shown.

to simultaneously fit the observed Stokes $(I, Q / I)$ profiles at all the limb distances. They also tried an appropriate combination of the FAL models to construct a combined model $(\overline{\mathrm{FALA}}+\mathrm{FALX})$. The combined model though provided a fit to the observed CLV of the $Q / I$, it failed to fit the CLV of the rest intensity and of the continuum intensity. They suggested that the next step would be to use the multi-dimensional modeling, which is computationally expensive. In this paper we attempt to simultaneously model the CLV of the $(I, Q / I)$ spectra using the FCHHT (Fontenla et al., 2009) solar 1-D model atmospheres. We expect that these kind of studies will help us to eliminate or justify the 1-D modeling approach before proceeding towards multi-dimensional modeling.

\section{Modeling Procedure}

The CLV observations of the Ca I $4227 \AA$ line used in this paper were obtained with the Zurich Imaging Polarimeter-3 (ZIMPOL-3) at IRSOL in Switzerland on October 16, 2012. Details of the observations and the stray light corrections to the observed $(I, Q / I)$ spectra are given in Supriya et al. (2014). The temperature structures of the FCHHT models used are shown in Figure 1. These models are more recent and updated versions of the FAL models. Along with the standard FCHHT models, the temperature structure of the combined model $\overline{\text { FALA }}+$ FALX is also shown. It was the $\overline{\text { FALA }}+$ FALX model that provided a fit to the observed $Q / I$ profiles at all limb distances (Supriya et al., 2014).

\subsection{CLV behavior using the FCHHT model atmospheres}

Figure 2 shows the angular dependence of the intensity (only at the line center) and the linear polarization at three chosen wavelength positions. The theoretical CLV profiles are computed using the standard FCHHT model atmospheres. Panel (a) of Figure 2 shows 

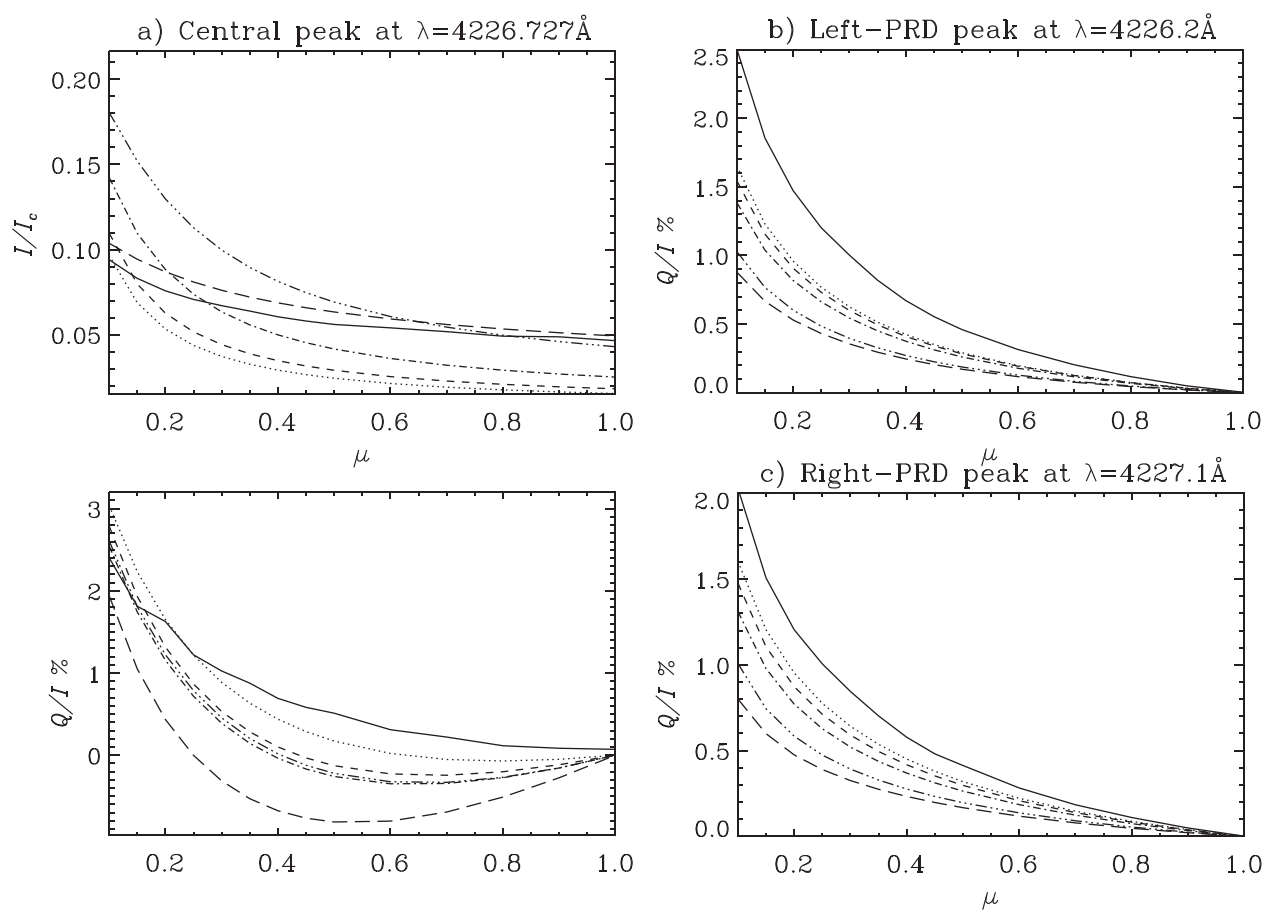

Figure 2. Observed (solid line) and calculated intensity and polarization signals as function of $\mu$ (observed at 14 points) at three different wavelength positions in the line profile. The theoretical spectra are calculated using standard models FCHHT-B (dotted line), FCHHT-D (dashed line), FCHHT-F (dot-dashed line), FCHHT-H (dash-triple-dotted line) and FCHHT-P (long dashed line).

a comparison between the observed and the theoretical CLV in $I$ and $Q / I$ at the line center wavelength. We see that the model FCHHT-P (long dashed line) is more suited for modeling the CLV of the line center intensity. But the same model is not suitable to reproduce the CLV of the observed $Q / I$ at the line center. Instead it is the model FCHHT-B that provides the closest fit to the CLV of the observed $Q / I$ at the line center. The model FCHHT-B also closely fits the CLV profiles of the $Q / I$ at the blue and red wing PRD peaks as seen from Figures 2 (b) and (c), respectively. Thus we do not find a single FCHHT atmospheric model which can provide a fit to the CLV of the Stokes $(I, Q / I)$ spectra simultaneously throughout the line profile.

\subsection{Comparison of the theoretical Stokes profiles from FCHHT-B and $\overline{\mathrm{FALA}}+\mathrm{FALX}$}

In Figure 3 we compare the observations with the theoretical profiles computed using the FCHHT-B model (which provides the closest fit among the FCHHT models) and also the $\overline{\text { FALA }}+$ FALX combined model. We see that the theoretical profiles computed using the $\overline{\text { FALA }}+$ FALX model indeed provides a better fit to the $Q / I$ profiles at different limb distances as compared to those computed using the FCHHT-B model atmosphere. It was pointed out in Supriya et al. (2014) that the $\overline{\text { FALA }}+$ FALX model is successful in providing a good fit to the $Q / I$ profiles at all limb distances, but it fails to fit the rest intensity. However from Figure 3 we see that the FCHHT-B model provides a good fit for the rest intensity at $\mu=0.1$, but it does not match the rest intensity for $\mu \geqslant$ 0.3. Also the FCHHT-B model only reproduces the shape of the observed $Q / I$ but does not provide a good fit to the CLV of the observed $Q / I$. From Figure 3 we see that the 

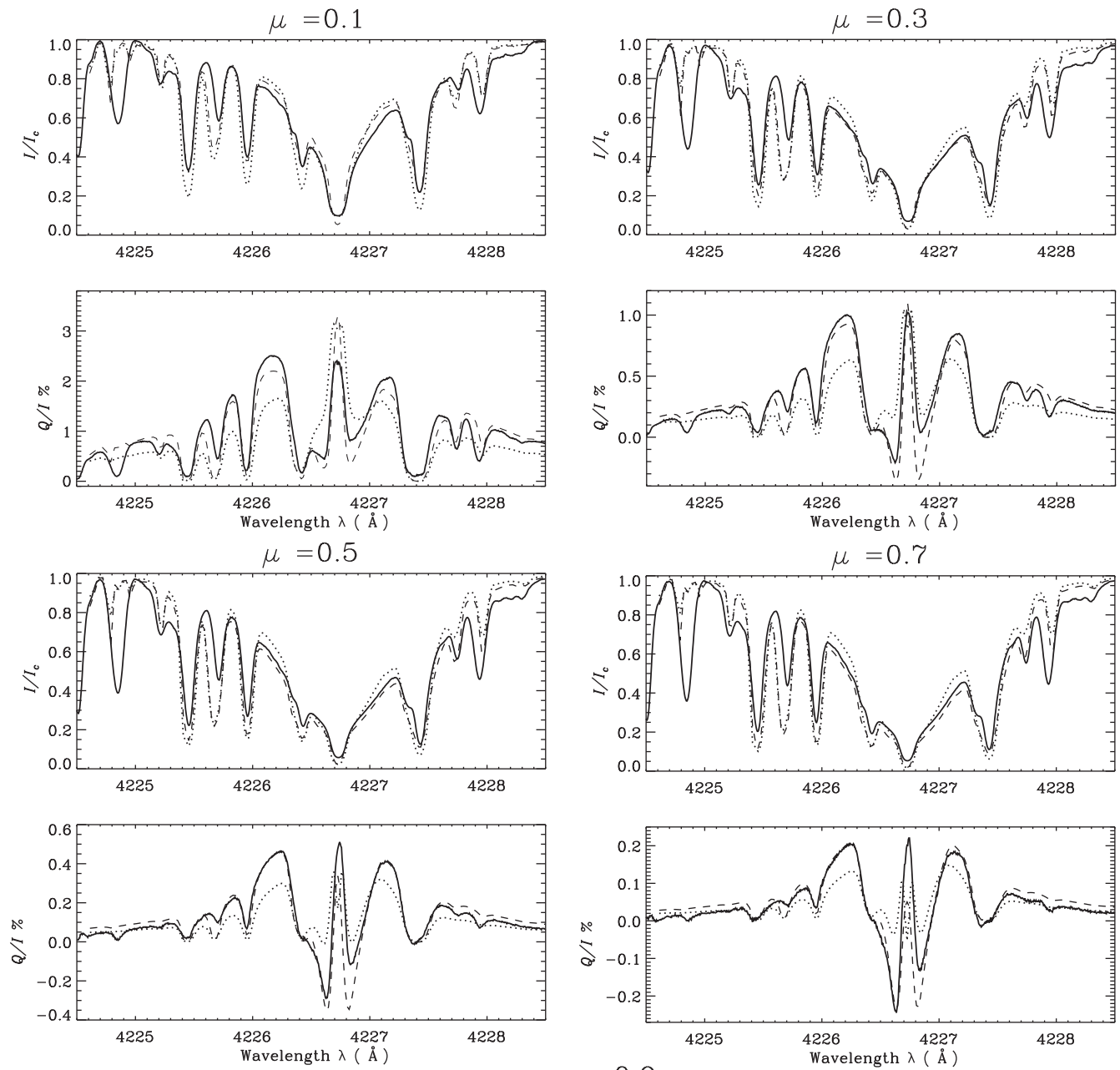

$\mu=0.9$
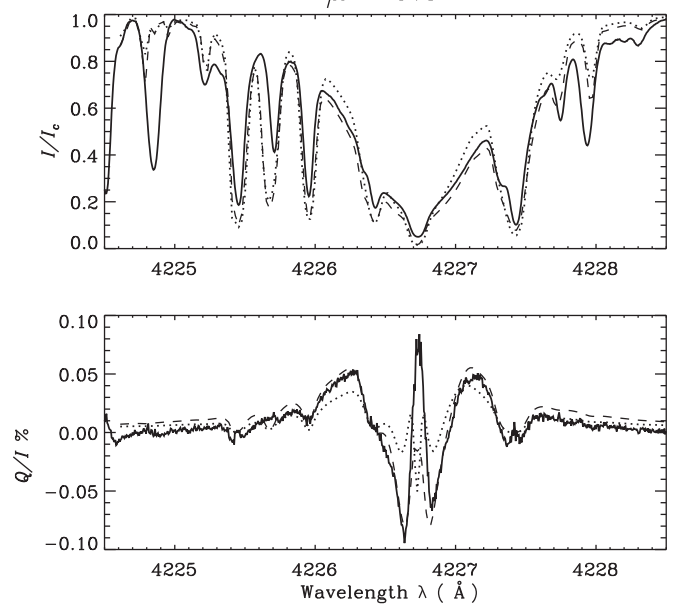

Figure 3. Comparison between the observed (solid line) and the theoretical Stokes profiles $(I, Q / I)$ at different limb distances. The theoretical profiles are computed using combined model $\overline{\text { FALA }}+$ FALX (dashed line) and FCHHT-B (dotted line). 


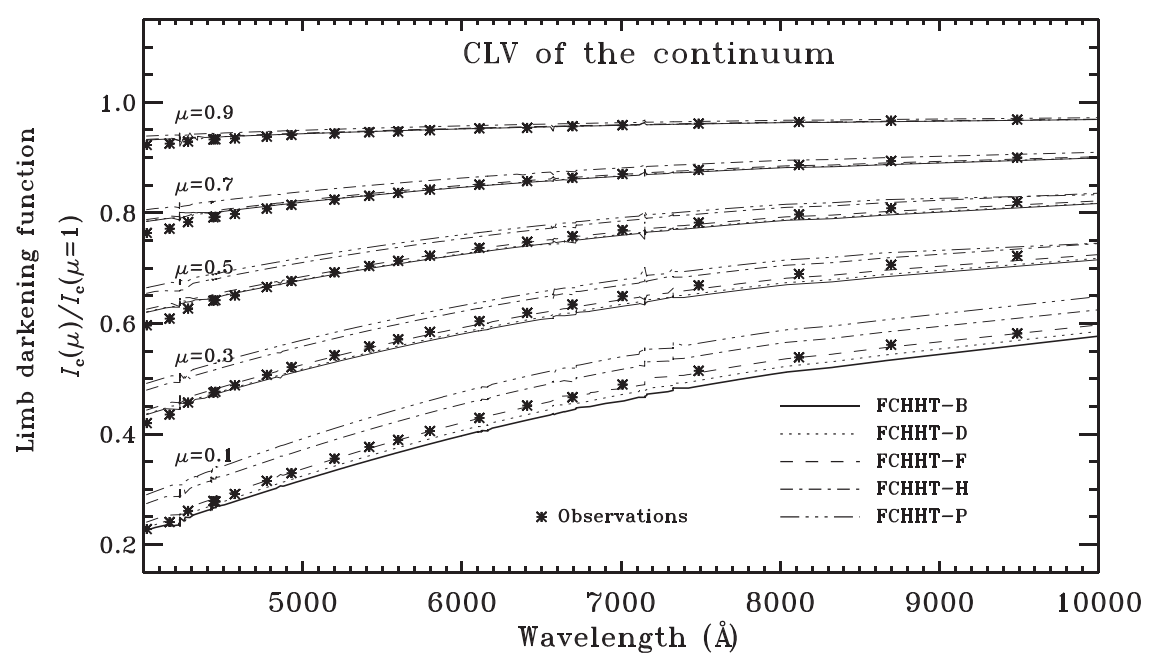

Figure 4. Comparison between the observed data for the CLV of continuum intensity from Neckel \& Labs (1994) for a range of wavelengths and the theoretical values from different FCHHT model atmospheres.

theoretical profiles computed using the FCHHT-B model atmosphere (dotted line) has a double peak structure in the $Q / I$ at the line center for $\mu>0.1$. We would like to note that this double peak cannot be smoothened even after appropriately smearing the theoretical profiles obtained using the FCHHT-B model, unlike in the case of the theoretical profiles obtained using the $\overline{\text { FALA }}+$ FALX model atmosphere. Next we examine the fit to the CLV of the continuum intensity over a wavelength range spanning from the visible to the infrared. Figure 4 shows the limb darkening function computed using the FCHHT model atmospheres for a range of wavelengths and $\mu$ values. The theoretical values from different models are compared with the observed data from Neckel \& Labs (1994). We see that the best fit to the observations is provided by the FCHHT-F model. The FCHHT-B model which provides closest fit to the CLV of the observed $Q / I$ does not provide the best fit to the observed CLV of the limb-darkening function. This leads us to the same conclusion as in Supriya et al. (2014) that it is indeed not possible to obtain a simultaneous fit to all the various observational constraints using single 1-D model atmosphere.

\section{Conclusions}

In this paper we have attempted to simultaneously model the center-to-limb variation of the $(I, Q / I)$ profiles of the Ca I $4227 \AA$ line using FCHHT model atmospheres. FCHHT model atmospheres are the recent and updated versions of the FAL model atmospheres. Our studies lead us to the conclusion that with 1-D model atmospheres it is not possible to obtain a simultaneous fit to all the observational constraints and thus a 1-D model cannot completely represent the actual solar atmosphere. The failure of FCHHT models to provide a simultaneous fit to the emergent $(I, Q / I)$ profiles at all the limb distances also substantiates this conclusion by Supriya et al. (2014). It is therefore necessary to go beyond 1-D modeling approach.

\section{Acknowledgements}

We are grateful to Dr. Han Uitenbroek for providing us with his realistic atmospheric modeling code. We acknowledge the use of the HYDRA cluster at the Indian Institute of 
Astrophysics for computations in this work. H.D.S. thanks COST ACTION MP1104 for the financial support provided to visit IRSOL. Research at IRSOL is financially supported by State Secretariat for Education, Research and Innovation, SERI, Canton Ticino, the city of Locarno, the local municipalities, and the Swiss National Science Foundation grant 200020-157103.

\section{References}

Fontela, J. M., Avrett, E. H., \& Loeser, R., 1993, ApJ 406, 319

Fontenla, J. M., Curdt, W., Haberreiter, M., Harder, J., \& Tian, H. 2009, ApJ 707, 482

Neckel, H. \& Labs, D., 1994, Solar Phys. 153, 91

Supriya, H. D., Smitha, H. N., Nagendra, K. N., Stenflo, J. O., Bianda, M., Ramelli, R., Ravindra, B., \& Anusha, L. S. 2014, ApJ 793, 42 